\title{
Structural Basis for the Retroreduction of Inactivated Peroxiredoxins by Human Sulfiredoxin,,$+ \neq$
}

\author{
Thomas J. Jönsson, Michael S. Murray, Lynnette C. Johnson, Leslie B. Poole, and W. Todd \\ Lowther \\ Center for Structural Biology, Department of Biochemistry, Wake Forest University School of \\ Medicine, Medical Center Boulevard, Winston-Salem, North Carolina 27157
}

\begin{abstract}
Sufiredoxins (Srx) repair the inactivated forms of typical two-Cys peroxiredoxins (Prx) implicated in hydrogen peroxide-mediated cell signaling. The reduction of the cysteine sulfinic acid moiety within the active site of the Prx by Srx involves novel sulfur chemistry and the use of ATP and $\mathrm{Mg}^{2+}$. The $1.65 \AA$ crystal structure of human Srx (hSrx) exhibits a new protein fold and a unique nucleotide binding motif containing the Gly98-Cys99-His100-Arg101 sequence at the N-terminus of an a-helix. HPLC analysis of the reaction products has confirmed that the site of ATP cleavage is between the $\beta$ - and $\gamma$-phosphate groups. Cys 99 and the $\gamma$-phosphate of ATP, modeled within the active site of the $2.0 \AA$ ADP product complex structure, are adjacent to large surface depressions containing additional conserved residues. These features and the necessity for significant remodeling of the Prx structure suggest that the interactions between hSrx and typical two-Cys Prxs are specific. Moreover, the concave shape of the hSrx active site surface appears to be ideally suited to interacting with the convex surface of the toroidal Prx decamer.
\end{abstract}

The ubiquitous peroxiredoxins (Prxs) ${ }^{1}$ have garnered much attention given the roles of family members in the detoxification of peroxides and the regulation of hydrogen peroxidemediated cellular signaling. Work from several laboratories has shown that these seemingly disparate roles are interrelated despite the differences in peroxide concentrations that are involved. For example, the typical two-Cys Prxs reduce hydrogen peroxide, alkyl hydroperoxides, and peroxynitrite via two conserved cysteine (Cys) residues per active site located on different subunits of an obligate dimer (Figure 1) $(1,2)$. The "peroxidatic" Cys residue $\left(\mathrm{Cys}-\mathrm{S}_{\mathrm{P}} \mathrm{H}\right)$ attacks the $\mathrm{O}-\mathrm{O}$ bond of the substrate, producing the first product (e.g., $\mathrm{H}_{2} \mathrm{O}$ or $\left.\mathrm{ROH}\right)$ and an oxidized, sulfenic acid $\left(\mathrm{Cys}-\mathrm{S}_{\mathrm{P}} \mathrm{OH}\right)$ intermediate. During normal catalysis, the "resolving" Cys residue $\left(\mathrm{Cys}-\mathrm{S}_{\mathrm{R}} \mathrm{H}\right)$ from the adjacent subunit reacts with the sulfenic acid group to form a disulfide bond. Cellular thiol-containing redox donors such as thioredoxin (Trx) return the Prxs to the activated state through thiol-disulfide exchange reactions $(1,3)$. During a transient intracellular burst of peroxide or oxidative stress

\footnotetext{
${ }^{\dagger}$ This research was supported by funds from the Wake Forest University School of Medicine and NIH Grant R01GM50389 to L.B.P. ₹The coordinates and structure factors for hSrx and the hSrx-ADP complex have been deposited in the Protein Data Bank as entries $1 \mathrm{XW} 3$ and 1XW4.

(C) 2005 American Chemical Society

* To whom correspondence should be addressed. tlowther@wfubmc.edu. Telephone: (336) 716-7230. Fax: (336) 777-3242.. SUPPORTING INFORMATION AVAILABLE

Relationship between hSrx molecules within the crystal lattice (Figure 1). This material is available free of charge via the Internet at http://pubs.acs.org.

${ }^{1}$ Abbreviations: AMPPNP, adenylyl imidodiphosphate; $\mathrm{Cys}-\mathrm{SPH}$, peroxidatic Cys residue; $\mathrm{Cys}-\mathrm{S}_{\mathrm{R}} \mathrm{H}$, resolving Cys residue; CysSPOH, Cys sulfenic acid; ET-hSrx, engineered truncation of hSrx; FL-hSrx, full-length hSrx; GSH, glutathione; HEPES, 4-(2hydroxyethyl)piperazine-1-ethanesulfonic acid; hPrx2, human Prx2; Prx, peroxiredoxin; SeMet, selenomethionine; Srx, sulfiredoxin; hSrx, human sufiredoxin; Trx, thioredoxin; TT-hSrx, trypsin-truncated hSrx.
} 
conditions, the Cys- $\mathrm{S}_{\mathrm{P}}-\mathrm{OH}$ intermediate can react, however, with another molecule of peroxide to yield the inactive, sulfinic acid form $\left(\mathrm{Cys}-\mathrm{S}_{\mathrm{P}} \mathrm{O}_{2}{ }^{-}\right)$of the $\mathrm{Cys}-\mathrm{S}_{\mathrm{P}} \mathrm{H}$ residue (Figure 1) (4-7). The susceptibility of eukaryotic Prxs to this type of inactivation appears to stem from two conserved sequence and structural motifs in the vicinity of the active site. The lack of these features in most microorganisms may account, at least in part, for the differing biological roles of Prxs in various organisms (8).

The ability of the yeast protein sulfiredoxin (Srx1) to repair or retroreduce (a term coined by the Rabilloud group) the yeast two-Cys Prx called Tsa1 (9) was unexpected, as Cys sulfinic and sulfonic acid generation was considered to be biologically irreversible $(10,11)$. Purified Srx1 was able to reduce overoxidized Tsa1 in the presence of ATP and $\mathrm{Mg}^{2+}$ or $\mathrm{Mn}^{2+}(9)$. A reductant, either dithiothreitol or thioredoxin, was also required for the reduction of Tsa1$\mathrm{SO}_{2}^{-}$to the Tsa1-SH form. Given the requirement for ATP hydrolysis, generation of a sulfinic phosphoryl ester (Cys- $\left.\mathrm{S}_{\mathrm{P}} \mathrm{O}_{2} \mathrm{PO}_{3}{ }^{2-}\right)$ intermediate was proposed (Figure 1). The inactivity of the Cys84Ser mutant also led Toledano and co-workers to further hypothesize the nucleophilic attack of Cys84-SH of Srx1 on the phosphorylated intermediate, resulting in formation of a thiosulfinate bond (i.e., a disulfide mono-oxide, Prx-Cys-SPO-S-Cys-Srx). Resolution of this complex with a reductant (e.g., Trx or glutathione) in this proposed mechanism would then return both enzymes to their reduced states through putative PrxCys- $\mathrm{S}_{\mathrm{P}} \mathrm{OH}$ and Srx1-S-S-R intermediates.

In an effort to discern the molecular basis for the novel sulfur chemistry of Srx and its interactions with Prxs, we have determined the crystal structures of human Srx (hSrx) in complex with phosphate and in complex with ADP, a product of the reaction. The structures reveal a new protein fold and a novel nucleotide binding motif. Biochemical analysis has also confirmed the site of ATP cleavage during the first step of the catalytic reaction. The overall concave shape of the hSrx active site surface suggests that hSrx is ideally suited to interacting with the overoxidized, doughnut-like Prx decamer.

\section{MATERIALS AND METHODS}

\section{Human Srx Purification and Crystallization}

The full-length (FL-hSrx, residues 1-137) and engineered (ET-hSrx, residues 32-137) constructs of hSrx were overexpressed in C41(DE3) Escherichia coli using a modified form of the pET19 vector (Novagen, Madison, WI) that contained an N-terminal His tag fusion and an intervening PreScission protease (Amersham Biosciences, Piscataway, NJ) cleavage site. Upon elution of the protein from a nickel affinity resin, $2 \mathrm{mM}$ EDTA, $15 \mathrm{mM}$ DTT, and 10 units of PreScission protease per milligram were added sequentially and incubated overnight at $4{ }^{\circ} \mathrm{C}$. Removal of the His tag in each case was verified by mass spectrometry. The resulting proteins contained four additional residues (Gly-Pro-His-Met) at the Nterminus left over from the protease cleavage site. The proteins were further purified and buffer exchanged into $20 \mathrm{mM}$ Hepes (pH 7.5) and $100 \mathrm{mM} \mathrm{NaCl}$ by being passed through a HiLoad Superdex 75 gel filtration column. The proteins were concentrated, aliquoted, flashfrozen with liquid nitrogen, and stored at $-80^{\circ} \mathrm{C}$. FL-hSrx was treated with trypsin (1:50, w/ w) to generate the TT construct (TT-hSrx, residues 38-137). The Leu46Met, Leu49Met, Leu82Met triple mutant and the Cys99Ser mutant of ET-hSrx were generated using the QuikChange Site-directed Mutagenesis Kit from Stratagene and the proteins purified in the same manner as the wild-type protein. Selenomethionine (SeMet) was incorporated into the Met triple mutant by the growth of cells in minimal media and the repression of endogenous Met synthesis prior to induction (12). Crystals of the wild-type and SeMet forms of ET-hSrx were obtained by the vapor diffusion method. Equal volumes of protein $[14.5-16.7 \mathrm{mg} / \mathrm{mL}$ in $20 \mathrm{mM}$ Hepes (pH 7.5) and $100 \mathrm{mM} \mathrm{NaCl}$ with or without $5 \mathrm{mM} \mathrm{DTT}$ ] and well solutions [1 M sodium-potassium phosphate $(\mathrm{pH} 8.5)$ and 6-10\% (( \pm )-2-methyl-2,4-pentanediol] were 
mixed and incubated at $20{ }^{\circ} \mathrm{C}$ for 3-10 days as sitting drops. Cryoprotectant [20\% ethylene glycol, $1 \mathrm{M}$ sodium-potassium phosphate ( $\mathrm{pH} 8.5)$, and 10\% (( \pm )-2-methyl-2,4-pentanediol] was slowly added to the crystal drop over a $6 \mathrm{~h}$ period. Cryoprotectant for the ADP-bound structure also contained $200 \mathrm{mM}$ ADP and $10 \mathrm{mM} \mathrm{MgCl}_{2}$.

\section{Data Collection and Structure Determination}

A three-wavelength MAD data set was collected on crystals of the Leu to Met variant of SeMet-labeled ET-hSrx on beamline X12C at the National Synchrotron Light Source (Upton, NY). The crystals exhibited $P 3_{2} 21$ symmetry $(a) 67.43 \AA, b$ ) $67.43 \AA$, and $c$ ) $51.07 \AA$ ) with one molecule in the asymmetric unit. Native and ADP data sets were collected on an in-house Rigaku/MSC MicroMax-007 generator with a Saturn-92 CCD detector. Data were merged and scaled with d*Trek (Rigaku/MSC, The Woodlands, TX). Three of the four potential selenium sites were found using SOLVE (13). The phases were improved by solvent flattening and maximum likelihood density modification with RESOLVE (13). The resulting 1.9 A electron density maps were unambiguous, and the autobuild feature of RESOLVE was able to generate $91 \%$ of the starting model. The chain trace and sequence register were independently verified by examining $1 \sigma$ and $5 \sigma$ experimental electron density maps generated by refining the SeMet sites with MLPHARE and solvent flattening with DM within the CCP4 program suite $(14,15)$. A comparison of the experimental and composite omit electron density maps facilitated the modification and extension of the model using O (16) and CNS (17). The SeMet-generated structure was used as the starting model for the molecular replacement solution of the native data set. The native structure required little rebuilding, but we did notice that Cys99, in contrast to the two other data sets, was not oxidized to the sulfinic acid. The native structure was used as the starting model for the ADP complex. At the initial stages of building for the ADP complex, the electron density for the ADP molecule was clearest upon examination of a $3 F_{\mathrm{o}}-2 F_{\mathrm{c}}$ map. The electron density for the ADP molecule gradually improved with refinement. All models were initially refined with CNS using alternating cycles of simulated annealing, positional, and $B$-factor refinement. The final cycles of refinement were performed with REFMAC5 (18). The final $R_{\text {work }}$ and $R_{\text {free }}$ values for the native $\left.\left(R_{\text {work }} / R_{\text {free }}\right) 21.4 \% / 25.8 \%\right)$ and $\left.\operatorname{ADP}\left(R_{\text {work }} / R_{\text {free }}\right) 21.8 \% / 27.1 \%\right)$ structures are only slightly elevated from the average values observed in the Protein Data Bank (18.5\%/23.0\% and 19.0\%/25.0\% for 1.6 and $2.0 \AA$ resolution, respectively) (19). Moreover, the $R_{\text {free }}$ values are similar to the expected values (26.4\% and $26.8 \%$ for the native and ADP structures, respectively) based on the reported correlation between the $R_{\mathrm{work}}$ and $R_{\mathrm{free}}$ values.

\section{Oxidation of Human Prx2, Derivatization of hSrx, and Analysis of Reaction Products}

Human Prx 2 was recombinantly expressed using the same vector system and expression strain that were used for hSrx. The N-terminal His tag was not removed after the nickel column step. The protein was further purified by being passed through anion exchange (Q Sepharose HP) and size exclusion columns (HiLoad Superdex 200). The protein $(20 \mu \mathrm{M})$ was oxidized to the sulfinic acid form by incubation (room temperature for $1 \mathrm{~h}$ ) with a 500fold molar excess of both $\mathrm{H}_{2} \mathrm{O}_{2}$ and dithiothreitol in 20 mM HEPES (pH 7.5) and $100 \mathrm{mM}$ $\mathrm{NaCl}$. This treatment causes redox cycling of the hPrx 2 with a fraction of the enzyme being oxidized to the sulfinic acid form during each cycle. The protein was passed back through the Superdex 200 column to remove the excess $\mathrm{H}_{2} \mathrm{O}_{2}$ and dithiothreitol. Complete oxidation of $h \operatorname{Prx} 2$ to the sulfinic acid form was confirmed by thiol quantitation via reaction with 5,5[.minute]-dithiobis(2-nitrobenzoic acid) (20) and mass spectrometry.

A covalently blocked form of Cys99 within ET-hSrx was generated by incubation of the thiol-containing protein with a 10-fold molar excess of iodoacetamide overnight on ice 
(addition of $\mathrm{CH}_{2} \mathrm{CONH}_{2}, 57 \mathrm{Da}$ ). Complete derivatization was confirmed by mass spectrometry.

The position of ATP cleavage was determined by eluting the reaction products from a Mono $\mathrm{Q}$ anion exchange column with a $30 \mathrm{~min}$ linear gradient $(1 \mathrm{~mL} / \mathrm{min})$ from 10 to $500 \mathrm{mM}$ $\mathrm{KH}_{2} \mathrm{PO}_{4}$, brought to $\mathrm{pH} 6$ with $\mathrm{KOH}$, with monitoring at $254 \mathrm{~nm}$. The AMP, ADP, and ATP standards were injected at concentrations of $500 \mu \mathrm{M}$ each. The sulfiredoxin reaction mixtures contained $25 \mathrm{mM}$ HEPES (pH 8.0), $100 \mathrm{mM} \mathrm{NaCl}, 500 \mu \mathrm{M}$ ATP, $1 \mathrm{mM} \mathrm{MgCl}{ }_{2}$, $25 \mu \mathrm{M}$ wild-type, Cys99Ser, or iodoacetamide-derivatized ET-hSrx, and $25 \mu \mathrm{M}$ overoxidized hPrx 2 . The reaction mixtures were incubated for $1 \mathrm{~h}$ at $37^{\circ} \mathrm{C}$. No ATP cleavage was observed for the reaction mixtures from which hSrx or hPrx 2 was omitted.

\section{RESULTS AND DISCUSSION}

\section{Protein Purification and Crystallization}

The full-length human Srx gene (residues 1-137) was cloned into an expression vector (pET19b derivative) that resulted in the addition of an $\mathrm{N}$-terminal poly-His tag followed by an engineered recognition sequence for the PreScission protease (Amersham Biosciences). Treatment of the recombinant hSrx with PreScission protease and dithiothreitol (DTT) released the affinity tag. An analysis of sequence alignments, however, suggested that the Nterminal, glycine-rich sequence (35\%; 13 of 37 residues) was unstructured and would prevent crystallization. On the basis of this analysis, the protein was also engineered with an $\mathrm{N}$-terminal truncation up to residue 32 [engineered truncation (ET-hSrx)]. Full-length hSrx was treated with trypsin in an effort to identify additional sequences at the $\mathrm{N}$ - and C-termini that may also need to be removed to facilitate crystallization. From the mass spectrometric analysis of the resulting fragments, the core domain of hSrx [trypsin truncation (TT-hSrx)] contains residues 38-137. Using this approach, 40-100 mg of the hSrx constructs was prepared and screened using the vapor diffusion method of crystallization. All three constructs exhibited similar activity (data not shown). Interestingly, only crystals of ET-hSrx could be obtained (see Materials and Methods). The largest crystals diffracted to $1.5 \AA$ resolution in house and were cryoprotected for data collection at $-170{ }^{\circ} \mathrm{C}$ by slow equilibration with a mother liquor containing $20 \%$ ethylene glycol.

\section{Overall Topology and Active Site}

The structure of ET-hSrx was determined using the multiwavelength anomalous dispersion (MAD) method and selenomethionine (SeMet) incorporation (21, 22). To use these methods, however, three conserved Leu residues (Leu36, Leu49, and Leu 82) were mutated to Met since the wild-type, recombinant protein contains only one Met residue left over from the PreScission protease cleavage site. This approach often does not affect protein structure and affords phasing (12). All three engineered SeMet sites were found during the phasing process using SOLVE (13). The $1.9 \AA$ MAD-phased experimental electron density map was unambiguous, and RESOLVE was able to autobuild $91 \%$ of the model. The chain trace and sequence register were independently verified as described in Materials and Methods.

The SeMet structure was used to determine the $1.65 \AA$ structure of the wild-type protein $\left[R_{\text {work }} / R_{\text {free }}\right.$ ) $21.4 \% / 25.8 \%$ (Table 1)] by molecular replacement (Figure 2 ). As briefly mentioned above, crystals could be obtained only for ET-hSrx. Analysis of the crystal contacts accounts for this phenomenon. Residues 29-37 project away from the core of the protein at a roughly $90^{\circ}$ angle to interact through 2-fold symmetry with residues 29-37 of another molecule (see the Supporting Information). Residues 54-59 from a third molecule add to these interactions, forming a three-stranded, antiparallel $\beta$-sheet. FL-hSrx and TT- 
hSrx would not be able to form this unusual packing due to steric restrictions and the loss of residues $29-37$, respectively.

A DALI (23) search of the available structure database showed that the structure of hSrx represents a new protein fold. The $\mathrm{N}$-terminus of the protein (Figure 2A) leads into a fivestranded, mixed $\beta$-sheet. Helix R2 packs against the $\beta$-sheet with its $\mathrm{N}$-terminus oriented toward the center of the protein. Helices R1 and R3 cap the ends of the structure. The preceding coil structure and helix R2 contain the signature sequence for Srxs, Phe96-Gly/ Ser97-Gly98-Cys99-His100-Arg101. This motif in the native structure coordinates a phosphate ion through multiple hydrogen bonding interactions (Figure 2B,C). Several water molecules (W1-W3) interact only with the phosphate ion.

The position of Cys99 at the N-terminus of an a-helix is reminiscent of many other proteins that use the a-helix dipole to facilitate biological activity and phosphate binding (reviewed in ref 24). For example, thioredoxin and its structural homologues, e.g., DsbA and glutaredoxin, all contain a CXXC motif where the first Cys residue is located at the Nterminus of the a-helix. The analysis of model peptides and a wide variety of site-directed mutants support the activation of the thiol group, i.e., the lowering of the $\mathrm{p} K_{\mathrm{a}}$ by several $\mathrm{pH}$ units (25-27). In other enzyme systems, e.g., papain and transglutaminase 3, the activated Cys residue is also part of a catalytic triad containing adjacent, noncontiguous His and Asp residues $(28,29)$. While there is no apparent biochemical or structural relationship between $\mathrm{hSrx}$ and these representative enzyme systems, the a-helix dipole motif most likely serves to activate Cys99 to the thiolate form (see the discussion below). Arg51, which is adjacent to Cys99 (NH2 atom-S ${ }^{\gamma}$ atom distance of $3.5 \AA$ ), may further activate Cys99 as the nucleophile of the reaction. This type of Arg-Cys interaction is also seen in Prxs and methionine sulfoxide reductase B $(2,30)$.

\section{ADP Complex, Novel Nucleotide Binding Motif, and Site of ATP Cleavage}

In an effort to determine the mode of binding of ATP to hSrx, the solution bathing the crystals of ET-hSrx was gradually exchanged with a synthetic mother liquor containing ethylene glycol and a potential ligand at $200 \mathrm{mM}$, either ATP, ADP, or the nonhydrolyzable ATP analogue adenylyl imidodiphosphate (AMPPNP). Data sets were collected and the structures determined by molecular replacement using the wild-type structure as the search model. Only the $2.0 \AA$ ADP complex structure (Table 1) showed convincing electron density for the nucleotide (Figure 3A). It is unclear why the electron density for the other nucleotides was not observed, even though one might expect ATP and its analogue AMPPNP to be more likely to displace the phosphate ion present in the active site of the native enzyme. A search for alternative crystallization conditions that do not include phosphate and cocrystallization experiments with the wild type and the inactive Cys99Ser mutant of ET-hSrx with ATP, AMPPNP, and ATP- $\gamma \mathrm{S}$ are ongoing.

As mentioned above, the $\beta$-phosphate group of ADP replaced the phosphate ion seen in the native and SeMet structures via similar interactions with His100 and Arg101 (Figure 3A). The occupancy of the ADP molecule was estimated with CNS to be 0.8 . This value is consistent with the slightly higher average $B$-factor value (Table 1 ) and the necessity to displace the phosphate ion found in the active site of the native enzyme structure. Lys61, also a conserved residue, was found to hydrogen bond to an oxygen atom of the a-phosphate group. The only other hydrogen bonds to ADP were from Ser64 and Thr68 to the adenine ring located within a shallow surface depression. A survey of the literature combined with the DALI search results supports the novelty of the Srx nucleotide-binding motif $(31,32)$.

The phosphate-nucleotide binding motif of hSrx does, however, show some similarity to protein tyrosine phosphatases and DNA ligases. For example, Srx and protein tyrosine 
phosphatases (e.g., PTP1B) both contain a conserved Cys residue adjacent to an Arg residue within a phosphate-binding motif. In PTP1B, however, the phosphate binding motif, (H/ $\mathrm{V}) \mathrm{CX}_{5} \mathrm{R}(\mathrm{S} / \mathrm{T})$, replaces the His100 of Srx with several main chain amide groups $(33,34)$. ATP- and NAD ${ }^{+}$-dependent DNA ligases connect two DNA strands through a process in which a conserved Lys residue that interacts with the a-phosphate group becomes adenylated $(35,36)$. The presence of the Lys61-a-phosphate interaction in hSrx (Figure 3A) prompted us to confirm the nature of ATP cleavage. ET-hSrx was incubated with the sulfinic acid form of recombinant $\mathrm{hPrx} 2$ in the presence of ATP and $\mathrm{Mg}^{2+}$ (Figure 3B). The chromatographic separation of the adenosine nucleotide standards and the Srx reaction products indicated that cleavage occurred at the $\beta-\gamma$ phosphodiester bond and not the $a-\beta$ bond. This observation is the most reasonable given the proximity of the $\gamma$-phosphate to Cys99 and the observed loss of activity when the yeast equivalent of Cys99 was mutated to Ser (9). Moreover, His 100 is appropriately placed to stabilize the developing charge on the bridging oxygen atom (Figure 3A) (37). To determine whether ATP hydrolysis was dependent on Cys99, the same reactions were performed with the Cys99Ser mutant of EThSrx and wild-type ET-hSrx pretreated with iodoacetamide. The alkylation and mutation of Cys99 significantly inhibited ATP hydrolysis, suggesting that Cys is involved in this step of the reaction. It may be, however, that even the small alkyl group added to the Cys99 residue or the subtle structural changes in the vicinity of the mutation are significant enough not to allow the Cys- $\mathrm{SO}_{2}{ }^{-}$group of the Prx molecule to approach the $\gamma$-phosphate of ATP.

The electron density in the ADP-complexed form of hSrx also showed that Cys99 had been oxidized to the sulfinic acid form (Csd99, Figure 3A), enabling a direct hydrogen bonding and salt bridge interaction with Arg51. The over-oxidation of Cys99 was also observed in the SeMet crystals. In contrast to the native structure which was collected on a freshly grown crystal, the latter structures were determined from older crystals. This observation is similar to that found for DJ-1 (38). It is unclear at this time whether the oxidation of Cys99 in hSrx has any biological relevance.

\section{Model of the ATP Complex and Putative Interactions with the Prx Decamer}

The hSrx-ADP structure was used to generate an ATP complex model (Figures 4A and 5). The $\gamma$-phosphate group could only be added to the ADP molecule in one orientation due to stereochemical constraints and potential collisions with active site residues and the other phosphate oxygen atoms. In this model, the $\gamma$-phosphate "propeller" makes a hydrogen bonding interaction with the backbone nitrogen atom of Gly98 and is positioned above Cys99 which is located at the bottom of a surface groove. Adjacent to the novel nucleotide binding site are two other pockets that presumably provide contacts to the macromolecular substrate of hSrx, the Prx doughnut-like decamer $(1,2)$. The smaller of the pockets is proximal to Cys99 and is lined by residues Arg51-Pro59. This region is separated from a significantly larger pocket by Gly97 and Gly 98 . Asp 80 is prominently positioned within the larger pocket lined by the side chains of Lys116, Tyr128, Asp124, Val127, and Phe96. These features and the coil structures near the active site of Srx (Figure 2A) may not be as flexible as one might expect on the basis of their surface location. This is in part due to the presence of multiple conserved Pro residues and hydrogen bonding interactions. On the basis of the previous evidence that mammalian and bacterial Prxs undergo dramatic structural changes during catalysis $(2,39)$, it is possible that Srx may induce structural changes in the Prx decamer that it is repairing.

The overoxidation of $h \operatorname{Prx} 2$ results in the stabilization of the decameric form of the enzyme (40). The five dimers within the $\left(a_{2}\right)_{5}$ decamer are arranged such that the active sites are oriented toward the outside of the toroidal structure in a staggered fashion. A closer look at one hPrx2 active site (Figure 4B) reveals that the sulfinic acid form of Cys51 (Csd51, Cys- 
$\mathrm{S}_{\mathrm{P}} \mathrm{O}_{2}^{-}$), located on the $\mathrm{N}$-terminal end of a a-helix, interacts with a conserved residue, Arg127. As described by Wood et al. (8), the GGLG and YF motifs are present only in peroxide-sensitive, typical two-Cys Prxs, including human Prx1-Prx4. These features are thought to slow the ability of the Cys- $\mathrm{S}_{\mathrm{R}} \mathrm{H}$ residue from the adjacent subunit of the dimer (>13 $\mathrm{A}$ away, Cys172- $\mathrm{S}_{\mathrm{R}} \mathrm{H}$ in hPrx2) to form a disulfide bond with the $\mathrm{Cys}-\mathrm{S}_{\mathrm{P}} \mathrm{OH}$ intermediate during the Prx reaction cycle (Figure 1). Therefore, the sulfenic acid intermediate has more opportunity to react with another substrate molecule, leading to formation of the Cys sulfinic acid and Prx inactivation.

While the unique sequence and structural features of eukaryotic, typical two-Cys Prxs support the molecular basis for peroxide sensitivity and inactivation, they may also impede access to the Cys- $\mathrm{S}_{\mathrm{P}} \mathrm{O}_{2}{ }^{-}$group that is to be repaired by Srx. Given the dimensions of $\mathrm{hSrx}$ [ $25 \AA 25 \AA 50 \AA$ (Figure 4A)], the symmetry and dimensions of the hPrx $2 \operatorname{dimer}(\sim 50 \AA x$ $50 \AA \times 60 \AA$ ), and the known compensatory changes that occur in typical two-Cys Prxs during catalysis $(2,39)$, a model for how one Srx molecule could bind to each Prx chain of the dimer (Figure 4C) was generated. The top panel in Figure 4C illustrates an "edge-on" view of four subunits of the Prx decamer with the obligate dimer shown in the middle. As mentioned above, the Prx active sites are oriented to the periphery of the decamer. Human Srx could "dock" to the circumference of the Prx doughnut like a belt (Figure 4C, bottom). The presence of the noncrystallographic, 2-fold symmetry axis supports the idea that the Srx molecules would have to bind such that their narrow dimensions are adjacent to each other in an antiparallel fashion, with the $\alpha 1$ and $\alpha 3$ helices functioning as molecular calipers. It is unclear whether the Srx molecules will contact each other and adjacent Prx dimers.

An analysis of the putative interface suggests that the displacement of the a-helix containing the Prx YF motif and potentially the GGLG motif are compulsory. The Prx a-helix containing the sulfinic acid group most likely also unwinds as observed in other Prx structures (2). While it is premature to consider the specific details of the Srx-Prx interactions without a structure of the complex, Asp80 of hSrx (Figure 4A) may play a key role in the remodeling of the Prx surface so that Cys99 of hSrx can gain access to the buried Cys- $\mathrm{S}_{\mathrm{P}} \mathrm{O}_{2}{ }^{-}$moiety. It is tempting to speculate that Asp80 disrupts the stable Cys sulfinic acid-Arg interaction [Csd51-Arg127 (Figure 4B)] of hPrx2 by forming an interaction with Arg127. This interaction may facilitate the local unfolding of the helix containing the Cys$\mathrm{S}_{\mathrm{P}} \mathrm{O}_{2}{ }^{-}$moiety. Additional residues on the surface of the Prx molecule may be important for Srx binding and catalysis. Chief among these are residues that may interact with the unsatisfied hydrogen-bonding sites of the ATP ribose ring and a-phosphate oxygen atoms (Figure 3A) and the small pocket adjacent to Cys99 generated by residues Pro52-Pro59 (Figure 4A).

\section{Putative Roles of Srx Residues in the Reaction Mechanism}

The crystal structures of hSrx presented herein and the biochemical data from the yeast (9) and human Srxs $(41,42)$ suggest that the reaction begins with the attack of the Cys- $\mathrm{S}_{\mathrm{P}} \mathrm{O}_{2}{ }^{-}$ group on the $\gamma$-phosphate of ATP (Figure 5). As described above, however, considerable structural rearrangements most likely take place in the Prx for this step to occur. His100 and Arg101 of the novel nucleotide binding motif, Lys61 (Figure 2B), and potentially other residues at the Prx-Srx macromolecular interface hold the ATP molecule in the correct register for catalysis. His 100 most likely functions to stabilize the developing negative charge on the oxygen atom that bridges the $\beta$ - and $\gamma$-phosphates. While the nucleotide binding motif of hSrx does remotely resemble the phosphate binding motif of protein tyrosine phosphates, the phosphorylation of Srx as the first step of the reaction seems unlikely given the need to activate the stable sulfinic acid group. This latter possibility, however, cannot be ruled out at this time. 
Human Srx can also use GTP, dGTP, or dATP as the phosphate donor in the reaction (41). This is consistent with the hSrx structure as it can accommodate the functional group differences between ATP and GTP. The ability of hSrx to use the deoxyribose form of the nucleotides also suggests that an interaction with the $2^{\prime}$-oxygen atom is not essential for positioning the ATP molecule for catalysis or mediating the Srx-Prx interface. The role of $\mathrm{Mg}^{2+}$ in the reaction is not apparent since it was not observed in the ADP crystal structure even though $10 \mathrm{mM} \mathrm{MgCl}_{2}$ was present during the crystal soaking experiment.

In the Srx mechanism proposed by the Toledano group (Figure 1), the putative attack on the ATP molecule leads to the formation of a sulfinic acid phosphoryl ester on Prx. This activated intermediate is reminiscent of the activation of a carboxyl group by ATP in a variety of enzyme systems (e.g., glutamine synthase, glutathione synthase, b-Ala:D-Ala ligase, and the peptidoglycan synthetases MurC/MurD) (43-45). In these systems, the acyl phosphate intermediate is broken down by the addition of either ammonia or the amine of an amino acid. The observation of DTT-reducible covalent complexes between the yeast Prxs and Srx1 (9) suggests that the activated sulfinic phosphoryl ester intermediate in the Srx reaction is broken down by the nucleophilic attack of the sulfur atom of Cys99 of hSrx. The hSrx crystal structures suggest that the $\mathrm{p} K_{\mathrm{a}}$ value of Cys 99 is lowered by the a-helix dipole and an interaction with Arg51. This proposal is consistent with the $\mathrm{p} K_{\mathrm{a}}$ of $\sim 7.3$ for hSrx as determined by an iodoacetamide titration (41). On the basis of the observation that DTT, glutathione (GSH), and Trx can support the reaction $(9,41)$, the resulting thiosulfinate (a cystine-S-monoxide) intermediate is broken down by the attack of a thiol on the Cys 99 sulfur atom. How GSH and Trx access the buried Cys99 residue in hSrx and in particular the putative Srx-Prx complex is not obvious. Therefore, the apparent inefficiency of hSrx (41) may be a consequence of the structural rearrangements needed to access the sulfinic acid group at the beginning of the reaction, to release Srx from the Srx-Prx intermolecular complex at the end of the reaction, or both.

\section{Biological Implications for Cys- $\mathrm{SO}_{2}-$ Formation and Repair}

Although this area is new to the field of protein repair, there is already some evidence accumulating that Cys sulfinic acid formation and repair in proteins is not uncommon (11). Indeed, several examples point to functional roles for Cys sulfinic acid formation in the activation of nitrile hydratase (46) and matrix metalloproteinases $(47,48)$. The Parkinson's disease protein DJ-1 is thought to be neuroprotective as a consequence of its Cys sulfinic acid-driven mitochondrial targeting (49). Cys sulfinic acid undoubtedly has additional roles in redox regulation, as well, although only a few in vitro examples exist so far [e.g., glutathione reductase inhibited by treatment with $\mathrm{NO}$ carriers (50), and the neutrophil S100A8 protein treated with hypochlorite (51)]. These observations suggest that Cys sulfinic acid formation in proteins may not just be inhibitory by removing thiols but rather can modulate reactivity in a more direct, functional way (52).

While the previous discussion may lead one to believe that hSrx may be a general protein repair enzyme, the new crystal structures presented herein and the biochemical data from the Rhee laboratory suggest the contrary. The novel fold of hSrx generates an extended active site with large surface depressions near the catalytic Cys99 residue (Figure 4A). These surface features, the discrete placement of conserved residues, and the apparent need for structural changes within the Prx substrate suggest that the interactions of hSrx with typical two-Cys Prxs (Prx1-Prx4) are specific. This view is supported by the inability of hSrx to repair oxidized human Prx5 (an atypical two-Cys Prx), Prx6 (a one-Cys Prx), and glyceraldehyde-3-phosphate dehydrogenase (GAPDH) (42). Moreover, hSrx was not able to repair Cys sulfinic acid of the yeast typical two-Cys Prx Tsa1, which is repaired by yeast Srx1, suggesting that species-specific interactions may also be present. The unique 17-amino 
acid insert found in yeast $\operatorname{Srx}$, between $\alpha 1$ and $\beta 3$ of hSrx, may be partly responsible for the specific interaction between ySrx and Tsa1.

It will be intriguing to compare the substrate specificity of hSrx with those of the sestrins (Hi95 and PA26) (53). The sestrins have also been shown to reduce Cys sulfinic acid in a Cys-, ATP-, and $\mathrm{Mg}^{2+}$-dependent manner. The sestrins, however, are significantly larger $(\sim 60 \mathrm{kDa})$ than the Srxs and show no apparent sequence homology. Thus, the sestrins may either preferentially repair the typical two-Cys Prxs with currently unknown structural and biochemical motifs or represent a more general repair enzyme. The lack of sestrins within yeast, however, suggests that Srx in this organism may have additional substrates.

\section{CONCLUSION}

Human Srx exhibits a new protein fold and a novel nucleotide binding motif containing the consensus Gly-Cys-His-Arg sequence. In the first step of the reaction, the ATP molecule is cleaved between the $\beta$ - and $\gamma$-phosphate groups. The $\gamma$-phosphate of ATP and the essential active site Cys residue are located adjacent to large surface invaginations containing additional conserved residues. These surface features and the known conformational flexibility of the Prxs suggest that specific interactions are made between hSrx and typical two-Cys Prxs. The overall concave shape of the hSrx active site surface also suggests that the repair protein binds to the convex perimeter of the Prx decamer. Additional interactions with adjacent subunits within the decamer may further stabilize the macromolecular interactions.

\section{Supplementary Material}

Refer to Web version on PubMed Central for supplementary material.

\section{Acknowledgments}

We are extremely grateful to Jamie Wallen and Dr. Conn Mallett of WFUSM and the NSLS X12C beamline staff for assistance in data collection and Mark Morris of the Wake Forest University Protein Analysis Core Lab (Winston-Salem, NC) for assistance with the HPLC analyses.

\section{REFERENCES}

1. Hofmann B, Hecht HJ, Flohé L. Peroxiredoxins. Biol. Chem. 2002; 383:347-364. [PubMed: 12033427]

2. Wood ZA, Schröder E, Harris JR, Poole LB. Structure, mechanism and regulation of peroxiredoxins. Trends Biochem. Sci. 2003; 28:32-40. [PubMed: 12517450]

3. Chae HZ, Kim HJ, Kang SW, Rhee SG. Characterization of three isoforms of mammalian peroxiredoxin that reduce peroxides in the presence of thioredoxin. Diabetes Res. Clin. Pract. 1999; 45:101-112. [PubMed: 10588361]

4. Mitsumoto A, Takanezawa Y, Okawa K, Iwamatsu A, Nakagawa Y. Variants of peroxiredoxins expression in response to hydroperoxide stress. Free Radical Biol. Med. 2001; 30:625-635. [PubMed: 11295360]

5. Chevallet M, Wagner E, Luche S, van Dorsselaer A, Leize-Wagner E, Rabilloud T. Regeneration of peroxiredoxins during recovery after oxidative stress: Only some over-oxidized peroxiredoxins can be reduced during recovery after oxidative stress. J. Biol. Chem. 2003; 278:37146-37153. [PubMed: 12853451]

6. Rabilloud T, Heller M, Gasnier F, Luche S, Rey C, Aebersold R, Benahmed M, Louisot P, Lunardi J. Proteomics analysis of cellular response to oxidative stress. Evidence for in vivo overoxidation of peroxiredoxins at their active site. J. Biol. Chem. 2002; 277:19396-19401. [PubMed: 11904290] 
7. Woo HA, Chae HZ, Hwang SC, Yang KS, Kang SW, Kim K, Rhee SG. Reversing the inactivation of peroxiredoxins caused by cysteine sulfinic acid formation. Science. 2003; 300:653-656. [PubMed: 12714748]

8. Wood ZA, Poole LB, Karplus PA. Peroxiredoxin evolution and the regulation of hydrogen peroxide signaling. Science. 2003; 300:650-653. [PubMed: 12714747]

9. Biteau B, Labarre J, Toledano MB. ATP-dependent reduction of cysteine-sulphinic acid by S. cereVisiae sulphiredoxin. Nature. 2003; 425:980-984. [PubMed: 14586471]

10. Claiborne A, Yeh JI, Mallett TC, Luba J, Crane EJ III, Charrier V, Parsonage D. Protein-sulfenic acids: Diverse roles for an unlikely player in enzyme catalysis and redox regulation. Biochemistry. 1999; 38:15407-15416. [PubMed: 10569923]

11. Hamann M, Zhang T, Hendrich S, Thomas JA. Quantitation of protein sulfinic and sulfonic acid, irreversibly oxidized protein cysteine sites in cellular proteins. Methods Enzymol. 2002; 348:146156. [PubMed: 11885268]

12. Gassner NC, Matthews BW. Use of differentially substituted selenomethionine proteins in X-ray structure determination. Acta Crystallogr. 1999; D55:1967-1970.

13. Terwilliger TC, Berendzen J. Automated MAD and MIR structure solution. Acta Crystallogr. 1999; D55:849-861.

14. Potterton E, Briggs P, Turkenburg M, Dodson E. A graphical user interface to the CCP4 program suite. Acta Crystallogr. 2003; D59:1131-1137.

15. Collaborative Computational Project Number 4. The CCP4 suite: Programs for protein crystallography. Acta Crystallogr. 1994; D50:760-763.

16. Jones TA, Zou JY, Cowan SW, Kjeldgaard M. Improved methods for building protein models in electron density maps and the location of errors in these models. Acta Crystallogr. 1991; A47:110119.

17. Brunger AT, Adams PD, Clore GM, DeLano WL, Gros P, Grosse-Kunstleve RW, Jiang JS, Kuszewski J, Nilges M, Pannu NS, Read RJ, Rice LM, Simonson T, Warren GL. Crystallography \& NMR system: A new software suite for macromolecular structure determination. Acta Crystallogr. 1998; D54:905-921.

18. Murshudov GN, Vagin AA, Dodson EJ. Refinement of macromolecular structures by the maximum-likelihood method. Acta Crystallogr. 1997; D53:240-255.

19. Kleywegt GJ, Jones TA. Homo crystallographicus: Quo vadis? Structure. 2002; 10:465-472. [PubMed: 11937051]

20. Poole LB. Flavin-dependent alkyl hydroperoxide reductase from Salmonella typhimurium. 2. Cystine disulfides involved in catalysis of peroxide reduction. Biochemistry. 1996; 35:65-75. [PubMed: 8555199]

21. Hendrickson WA, Ogata CM. Phase determinations from multiwavelength anomalous diffraction measurements. Methods Enzymol. 1997; 276:494-523.

22. Ealick SE. Advances in multiple wavelength anomalous diffraction crystallography. Curr. Opin. Chem. Biol. 2000; 4:495-499. [PubMed: 11006535]

23. Holm L, Sander C. Dali: A network tool for protein structure comparison. Trends Biochem. Sci. 1995; 20:478-480. [PubMed: 8578593]

24. Hol WG. The role of the R-helix dipole in protein function and structure. Prog. Biophys. Mol. Biol. 1985; 45:149-195. [PubMed: 3892583]

25. Kortemme T, Creighton TE. Ionisation of cysteine residues at the termini of model a-helical peptides. Relevance to unusual thiol $\mathrm{pK}_{\mathrm{a}}$ values in proteins of the thioredoxin family. J. Mol. Biol. 1995; 253:799-812. [PubMed: 7473753]

26. Mössner E, Huber-Wunderlich M, Glockshuber R. Characterization of Escherichia coli thioredoxin variants mimicking the active sites of other thiol-disulfide oxidoreductases. Protein Sci. 1998; 7:1233-1244. [PubMed: 9605329]

27. Philipps B, Glockshuber R. Randomization of the entire active-site helix R1 of the thiol-disulfide oxidoreductase DsbA from Escherichia coli. J. Biol. Chem. 2002; 277:43050-43057. [PubMed: 12193604] 
28. Ahvazi B, Boeshans KM, Idler W, Baxa U, Steinert PM, Rastinejad F. Structural basis for the coordinated regulation of transglutaminase 3 by guanine nucleotides and calcium/magnesium. $\mathrm{J}$. Biol. Chem. 2004; 279:7180-7192. [PubMed: 14645372]

29. Doran JD, Carey PR. R-Helix dipoles and catalysis: Absorption and Raman spectroscopic studies of acyl cysteine proteases. Biochemistry. 1996; 35:12495-12502. [PubMed: 8823185]

30. Lowther WT, Weissbach H, Etienne F, Brot N, Matthews BW. The mirrored methionine sulfoxide reductases of Neisseria gonorrhoeae pilB. Nat. Struct. Biol. 2002; 9:348-352. [PubMed: 11938352]

31. Traut TW. The functions and consensus motifs of nine types of peptide segments that form different types of nucleotide-binding sites. Eur. J. Biochem. 1994; 222:9-19. [PubMed: 8200357]

32. Saraste M, Sibbald PR, Wittinghofer A. The P-loop: A common motif in ATP- and GTP-binding proteins. Trends Biochem. Sci. 1990; 15:430-434. [PubMed: 2126155]

33. Pannifer AD, Flint AJ, Tonks NK, Barford D. Visualization of the cysteinyl-phosphate intermediate of a protein-tyrosine phosphatase by X-ray crystallography. J. Biol. Chem. 1998; 273:10454-10462. [PubMed: 9553104]

34. Peters GH, Frimurer TM, Olsen $\mathrm{OH}$. Electrostatic evaluation of the signature motif $(\mathrm{H} / \mathrm{V}) \mathrm{CX}_{5} \mathrm{R}(\mathrm{S} /$ T) in protein-tyrosine phosphatases. Biochemistry. 1998; 37:5383-5393. [PubMed: 9548920]

35. Timson DJ, Singleton MR, Wigley DB. DNA ligases in the repair and replication of DNA. Mutat. Res. 2000; 460:301-318. [PubMed: 10946235]

36. Martin IV, MacNeill SA. ATP-dependent DNA ligases. Genome Biol. 2002; 3:REVIEWS3005. [PubMed: 11983065]

37. Admiraal SJ, Herschlag D. Mapping the transition state for ATP hydrolysis: Implications for enzymatic catalysis. Chem. Biol. 1995; 2:729-739. [PubMed: 9383480]

38. Wilson MA, Collins JL, Hod Y, Ringe D, Petsko GA. The 1.1-Å resolution crystal structure of DJ-1, the protein mutated in autosomal recessive early onset Parkinson's disease. Proc. Natl. Acad. Sci. U.S.A. 2003; 100:9256-9261. [PubMed: 12855764]

39. Wood ZA, Poole LB, Hantgan RR, Karplus PA. Dimers to doughnuts: Redox-sensitive oligomerization of 2-cysteine peroxiredoxins. Biochemistry. 2002; 41:5493-5504. [PubMed: 11969410]

40. Schröder E, Littlechild JA, Lebedev AA, Errington N, Vagin AA, Isupov MN. Crystal structure of decameric 2-Cys peroxiredoxin from human erythrocytes at $1.7 \AA$ resolution. Structure. 2000; 8:605-615. [PubMed: 10873855]

41. Chang TS, Jeong W, Woo HA, Lee SM, Park S, Rhee SG. Characterization of mammalian sulfiredoxin and its reactivation of hyperoxidized peroxiredoxin through reduction of cysteine sulfinic acid in the active site to cysteine. J. Biol. Chem. 2004; 279:50994-51001. [PubMed: 15448164]

42. Woo HA, Jeong W, Chang TS, Park KJ, Park SJ, Yang JS, Rhee SG. Reduction of cysteine sulfinic acid by sulfiredoxin is specific to 2-Cys peroxiredoxins. J. Biol. Chem. 2004; 280:3125-3128. [PubMed: 15590625]

43. Bouhss A, Dementin S, van Heijenoort J, Parquet C, Blanot D. Formation of adenosine 5' tetraphosphate from the acyl phosphate intermediate: A difference between the MurC and MurD synthetases of Escherichia coli. FEBS Lett. 1999; 453:15-19. [PubMed: 10403366]

44. Fan C, Moews PC, Shi Y, Walsh CT, Knox JR. A common fold for peptide synthetases cleaving ATP to ADP: Glutathione synthetase and d-alanine:d-alanine ligase of Escherichia coli. Proc. Natl. Acad. Sci. U.S.A. 1995; 92:1172-1176. [PubMed: 7862655]

45. Eisenberg D, Gill HS, Pfluegl GM, Rotstein SH. Structure-function relationships of glutamine synthetases. Biochim. Biophys. Acta. 2000; 1477:122-145. [PubMed: 10708854]

46. Murakami T, Nojiri M, Nakayama H, Odaka M, Yohda M, Dohmae N, Takio K, Nagamune T, Endo I. Post-translational modification is essential for catalytic activity of nitrile hydratase. Protein Sci. 2000; 9:1024-1030. [PubMed: 10850812]

47. Fu X, Kassim SY, Parks WC, Heinecke JW. Hypochlorous acid oxygenates the cysteine switch domain of promatrilysin (MMP-7). A mechanism for matrix metalloproteinase activation and atherosclerotic plaque rupture by myeloperoxidase. J. Biol. Chem. 2001; 276:41279-41287. [PubMed: 11533038] 
48. Gu Z, Kaul M, Yan B, Kridel SJ, Cui J, Strongin A, Smith JW, Liddington RC, Lipton SA. SNitrosylation of matrix metalloproteinases: Signaling pathway to neuronal cell death. Science. 2002; 297:1186-1190. [PubMed: 12183632]

49. Canet-Aviles RM, Wilson MA, Miller DW, Ahmad R, McLendon C, Bandyopadhyay S, Baptista MJ, Ringe D, Petsko GA, Cookson MR. The Parkinson's disease protein DJ-1 is neuroprotective due to cysteine-sulfinic acid-driven mitochondrial localization. Proc. Natl. Acad. Sci. U.S.A. 2004; 101:9103-9108. [PubMed: 15181200]

50. Becker K, Savvides SN, Keese M, Schirmer RH, Karplus PA. Enzyme inactivation through sulfhydryl oxidation by physiologic NO-carriers. Nat. Struct. Biol. 1998; 5:267-271. [PubMed: 9546215]

51. Raftery MJ, Yang Z, Valenzuela SM, Geczy CL. Novel intra- and inter-molecular sulfinamide bonds in S100A8 produced by hypochlorite oxidation. J. Biol. Chem. 2001; 276:33393-33401. [PubMed: 11445563]

52. Okeley NM, van der Donk WA. Novel cofactors via post-translational modifications of enzyme active sites. Chem. Biol. 2000; 7:R159-R171. [PubMed: 10903941]

53. Budanov AV, Sablina AA, Feinstein E, Koonin EV, Chumakov PM. Regeneration of peroxiredoxins by p53-regulated sestrins, homologs of bacterial AhpD. Science. 2004; 304:596600. [PubMed: 15105503]

54. DeLano, WL. The PyMOL User's Manual. DeLano Scientific; San Carlos, CA: 2002. 


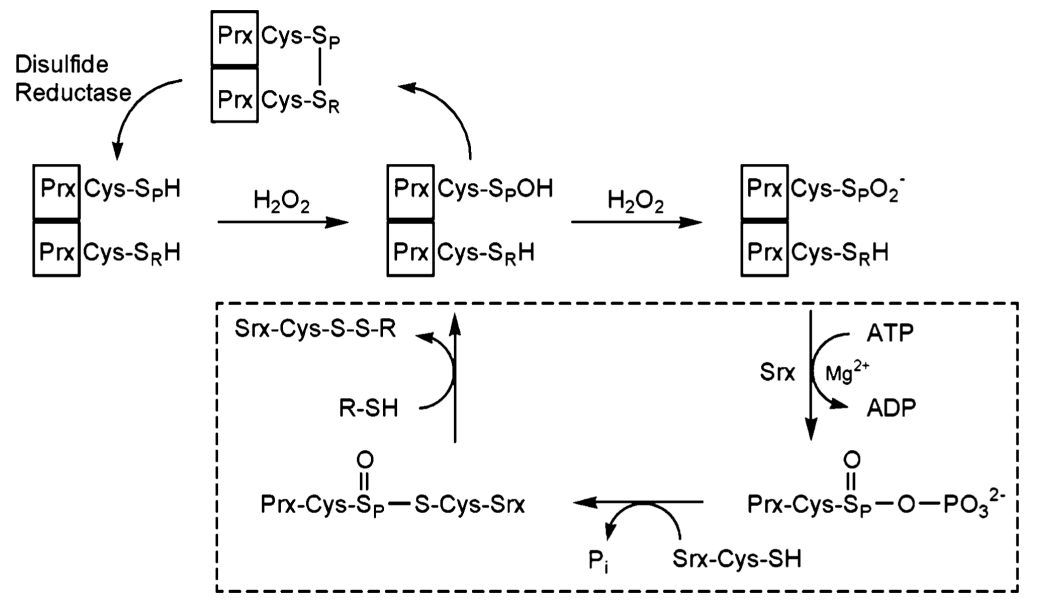

Figure 1.

Interrelationships between the catalytic and inactivation pathways of Prxs and the proposed mechanism of Srx. In the top part of the figure, the $\mathrm{Cys}-\mathrm{S}_{\mathrm{P}} \mathrm{H}$ and $\mathrm{Cys}-\mathrm{S}_{\mathrm{R}} \mathrm{H}$ residues of the Prx dimer (each from one subunit of the dimer; indicated by the divided rectangle) reduce peroxides, e.g., $\mathrm{H}_{2} \mathrm{O}_{2}$, through the formation of a sulfenic acid intermediate $(\mathrm{Cys}-\mathrm{S} \mathrm{OH})$ and an intermolecular disulfide bond which is ultimately reduced by a disulfide reductase $(1,2)$. Reaction of the Cys- $\mathrm{S}_{\mathrm{P}} \mathrm{OH}$ moiety with another molecule of substrate results in the formation of Cys sulfinic acid $\left(\mathrm{Cys}-\mathrm{S}_{\mathrm{P}} \mathrm{O}_{2}{ }^{-}\right)$and consequently the inactivation of the Prx molecule. The proposed mechanism of Srx (dashed box) begins with the activation of the sulfinic acid group by reaction with ATP (9). Additional steps include the thiol-dependent ( $\mathrm{R}-\mathrm{SH})$ resolution of a sulfinic phosphoryl ester $\left(\mathrm{Cys}-\mathrm{S}_{\mathrm{P}} \mathrm{O}_{2} \mathrm{PO}_{3}{ }^{2-}\right)$ and thiosulfinate (PrxCys-S $\mathrm{P}_{\mathrm{P}} \mathrm{O}$-SCys-Srx) intermediates. The second subunit of the Prx dimer and the Cys-S $\mathrm{R}$ residue are not shown in the Srx mechanism for clarity. See the text for further details. 

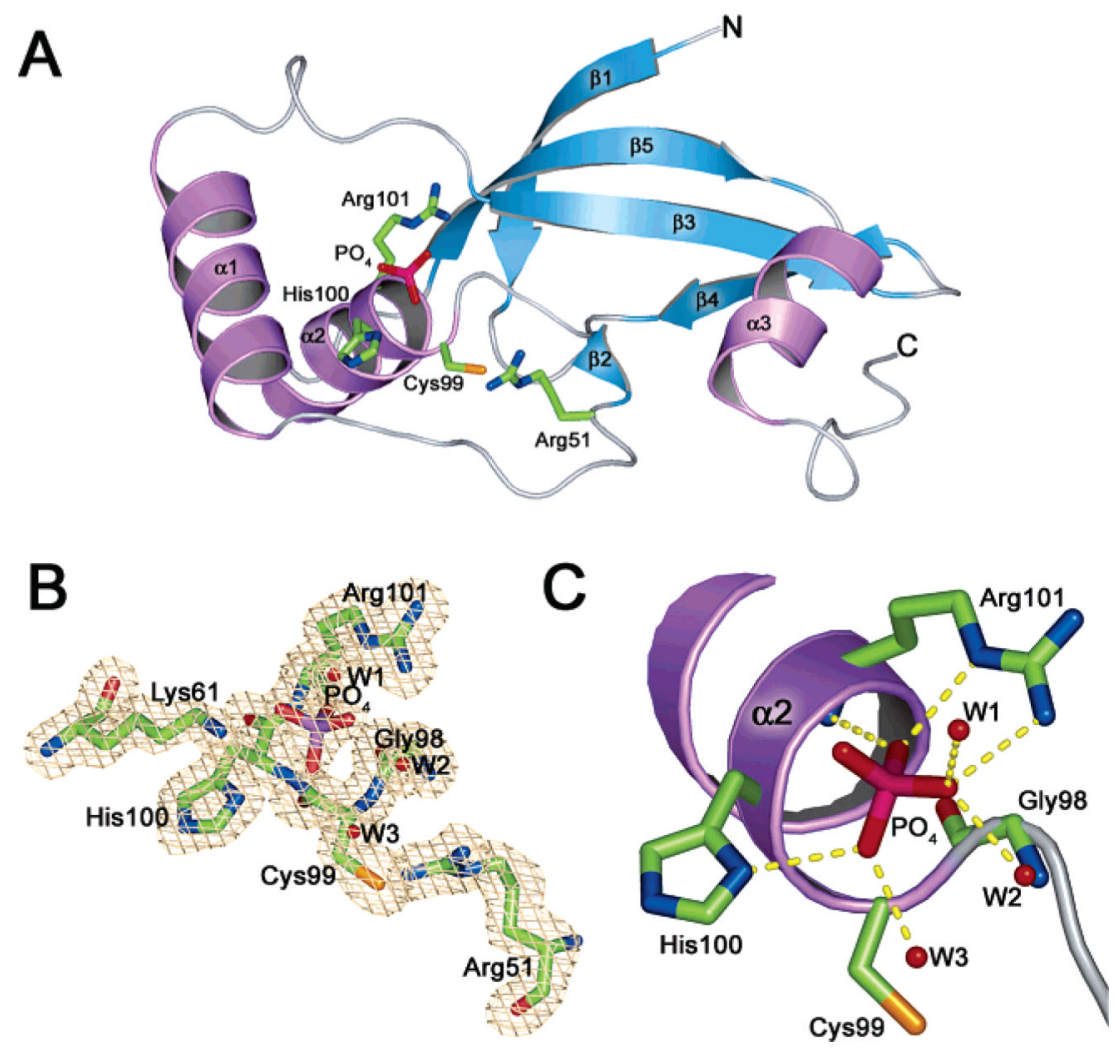

Figure 2.

Structure of human sulfiredoxin. (A) Overall fold of hSrx in complex with phosphate. The a-helices (pink) and $\beta$-strands (blue) are numbered consecutively on the basis of the primary sequence. The N-terminal extension (residues 28-36) that protrudes from the protein surface to establish an unusual crystal contact (see Figure 1 of the Supporting Information for details) and the 310 -helicies are not shown for clarity. Arg51, Cys99, His100, Arg101, and a phosphate molecule $\left(\mathrm{PO}_{4}\right)$ are shown as sticks to illustrate the relationship of the active site structures to the novel protein fold. Atom colors are as follows: green for carbon, blue for nitrogen, red for oxygen, orange for sulfur, and magenta for phosphate. (B) Electron density (simulated annealing, $F_{\mathrm{O}}-F_{\mathrm{c}}$ omit map contoured at $3.0 \sigma$ ) within the active site of the refined model of wild-type ET-hSrx. Three water molecules (W1-W3) that interact with the phosphate ion are colored red. (C) Molecular interactions among the conserved GCHR motif of hSrx, phosphate, and solvent. Putative hydrogen bonding interactions are shown as dashed yellow lines. The phosphate molecule is bound by interactions with His 100 and Arg101. The sulfur atom of the side chain of Cys99 is oriented outside the R2 helical axis and is $3.5 \AA$ from the $\mathrm{NH} 2$ atom of Arg51 (as shown in panels A and B). This figure and subsequent molecular images were generated with PYMOL (54). 

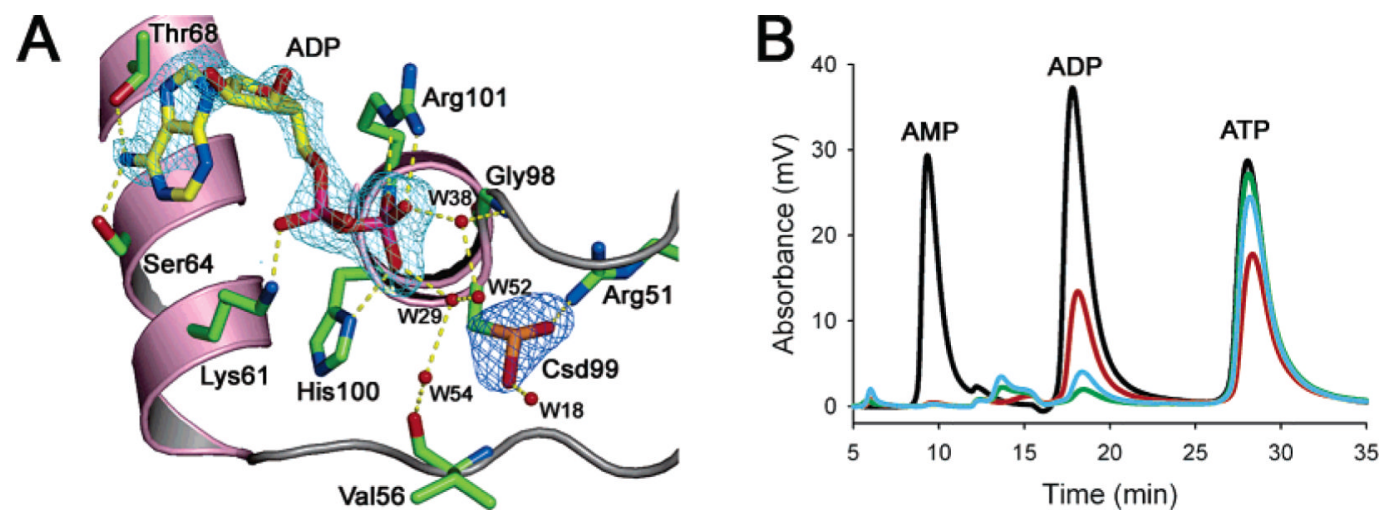

Figure 3.

Structure of ADP bound to hSrx and the site of ATP cleavage. (A) Electron density and molecular interactions among the enzyme, solvent, and ADP. Simulated annealing, $F_{\mathrm{o}}-F_{\mathrm{c}}$ omit maps are contoured at $2 \sigma$ and $4 \sigma$ for ADP (cyan) and the oxidized, sulfinic acid form of Cys99 (Csd99, blue), respectively. Putative hydrogen bonding interactions are shown as dashed yellow lines. The adenine ring of ADP interacts with Ser64 and Thr68. One of the oxygen atoms of the a-phosphate group interacts with Lys61. The oxygen atoms of the $\beta$ phosphate group interact with His100, Arg101, W29, and W38. The backbone nitrogen atom of Arg101 also interacts with the oxygen atom (O1B) of the $\beta$-phosphate (not shown). Additional hydrogen bonding interactions extend from water molecules W29 and W38 to include Gly98, W52, W54, and Val56. Csd99 forms interactions with the conserved Arg51 and W18. (B) HPLC analysis of the site of ATP cleavage during the Srx reaction. Nucleotide standards (black line) were eluted from the Mono Q anion exchange column (see Materials and Methods) with monitoring at $254 \mathrm{~nm}$. The Srx reaction was initiated by mixing wild-type, overoxidized hPrx2, wild-type ET-hSrx, ATP, and $\mathrm{MgCl}_{2}$ (red line). After incubation at $37^{\circ} \mathrm{C}$ for $1 \mathrm{~h}$, the samples were analyzed. Reactions were also carried out in which Cys99 of ET-hSrx was either prederivatized with iodoacetamide (green line) or mutated to Ser (cyan line). 

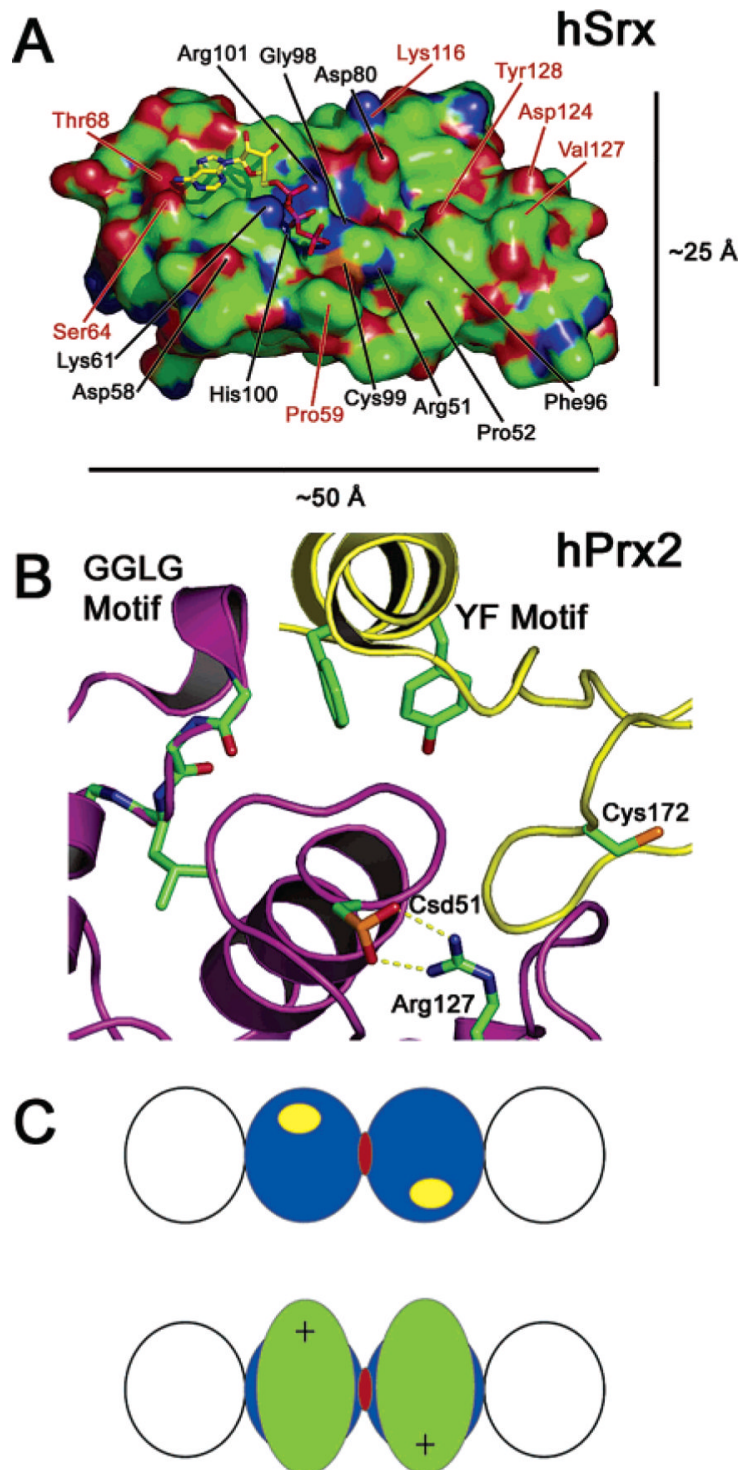

\section{Srx:Prx Model}

Figure 4.

hSrx surface features, hPrx 2 active site, and model for the Srx-Prx interaction. (A) Solvent contact surface representation (1.7 $\AA$ probe) of hSrx with ATP modeled within the active site. The atom colors are the same as in Figure 1. Residues highlighted with black lines are strictly conserved. Gly98, Cys99, His100, Arg101, and Lys61 mediate ATP binding. These residues along with Pro52, Asp58, Asp80, and Phe96 may also play a key role in establishing the Srx-Prx macromolecular interface. Other residues that may also contribute to the latter role are highlighted in red: Pro59, Ser64, Thr68, Lys116, Asp124, Val127, and Tyr128. (B) Close-up of the hPrx $2 a_{2}$ dimer (purple and yellow chains) on the perimeter of the sulfinic acid form of the hPrx 2 decamer (PDB entry 1QMV) (40). The hydrogen bonds between $\mathrm{Csd} 51\left(\mathrm{Cys} 51-\mathrm{S}_{\mathrm{P}} \mathrm{O}_{2}^{-}\right)$and $\mathrm{Arg} 127$ stabilize the active site. Nearby within the same chain is the Gly-Gly-Leu-Gly (GGLG) motif (residues 93-96). The Tyr-Phe (YF) motif within the C-terminal helix (residues 187-197) and the resolving Cys residue, Cys172, are contributed from the adjacent subunit of the dimer. (C) Model for the Srx-Prx interaction. The top panel is an edge-on view of four subunits of the Prx decamer before the binding of 
Srx. The $a_{2}$ dimer (blue) is shown in the center with the active sites and the noncrystallographic, 2-fold axis colored yellow and red, respectively. Adjacent Prx subunits within the decamer are represented by the nonfilled, black circles. The bottom panel shows Srx molecules (green) bound in an antiparallel manner to the circumference of the Prx ringlike decamer. Only the Srx molecules bound to the central Prx dimer are shown. The plus sign indicates directionality. The Srx molecules may interact with each other and adjacent Prx subunits. 


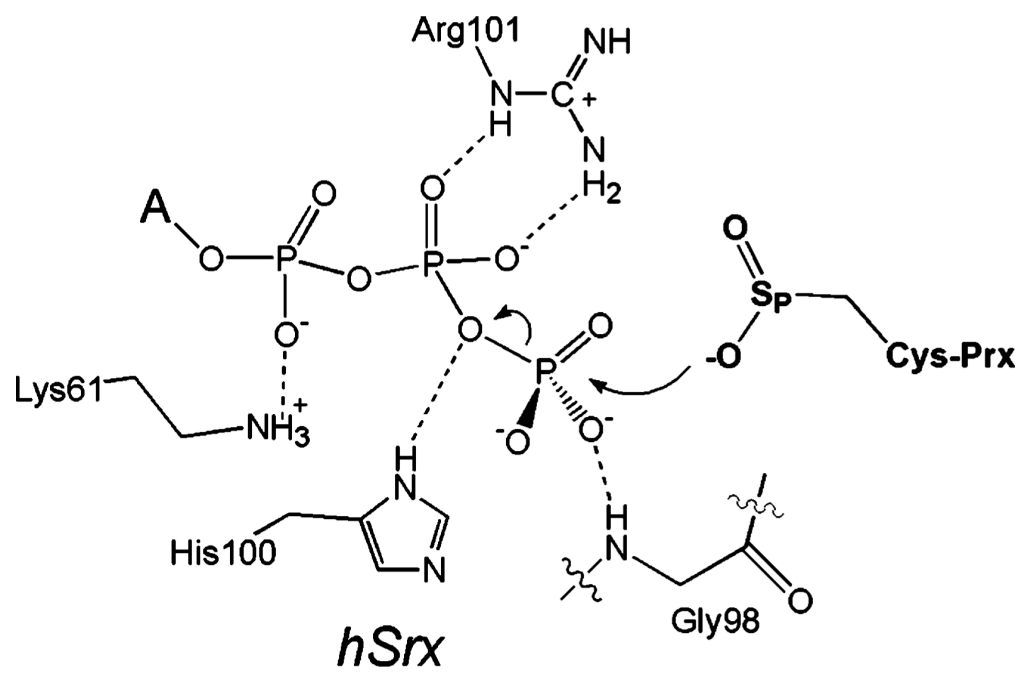

Figure 5.

Proposed mode of binding of ATP and the first step of the hSrx reaction. Putative hydrogen bonding interactions between hSrx and ATP (nucleotide and sugar structures denoted by A) are indicated by dashed lines. The Cys sulfinic acid moiety of the Prx molecule to be repaired is shown in bold. See the text for details. 
Table 1

Crystallographic Data, Phasing, and Refinement Statistics

\begin{tabular}{|c|c|c|c|c|c|}
\hline & \multicolumn{3}{|c|}{ SeMet L46,49,82M triple mutant } & \multirow{2}{*}{$\frac{\text { wild type }}{\text { native }}$} & \multirow{2}{*}{$\frac{\text { wild type }}{\text { ADP }}$} \\
\hline & peak & inflection & remote & & \\
\hline \multicolumn{6}{|c|}{ Crystallographic Data } \\
\hline wavelength $(\AA)$ & 0.9775 & 0.9778 & 0.9500 & 1.54 & 1.54 \\
\hline resolution range $(\AA)$ & $33.7-1.9$ & $38.5-1.9$ & $38.4-1.9$ & $38.7-1.65$ & $29.6-2.0$ \\
\hline no. of observed reflections & 231032 & 232125 & 230540 & 118194 & 92049 \\
\hline no. of unique reflections & 20516 & 20510 & 20507 & 16687 & 9562 \\
\hline$R_{\mathrm{sym}}^{a, b}(\%)$ & $9.0(29.9)$ & $8.9(28.4)$ & $9.0(29.6)$ & $4.3(23.0)$ & $5.1(28.8)$ \\
\hline completeness $^{a}(\%)$ & $100(100)$ & $100(100)$ & $100(100)$ & $98.9(92.2)$ & $99.6(95.3)$ \\
\hline$\langle I\rangle /\langle\sigma(I)\rangle^{a, c}$ & $18.4(5.8)$ & $18.8(5.9)$ & $18.0(5.6)$ & $24.3(4.1)$ & $30.9(4.4)$ \\
\hline \multicolumn{6}{|c|}{ Phasing } \\
\hline \multicolumn{6}{|l|}{ overall figure of merit } \\
\hline $\operatorname{SOLVE}^{a}$ & \multicolumn{3}{|c|}{$0.60(0.39)$} & & \\
\hline $\operatorname{RESOLVE}^{a}$ & \multicolumn{3}{|c|}{$0.73(0.43)$} & & \\
\hline \multicolumn{6}{|c|}{ Refinement } \\
\hline$R_{\text {work }}^{d}(\%)$ & & & & 21.4 & 21.8 \\
\hline$R_{\text {free }}^{e}(\%)$ & & & & 25.8 & 27.1 \\
\hline \multicolumn{6}{|l|}{ rms deviation } \\
\hline bond lengths ( $\mathrm{A})$ & & & & 0.02 & 0.02 \\
\hline bond angles (deg) & & & & 1.57 & 1.83 \\
\hline \multicolumn{6}{|l|}{ average $B$-factor $\left(\AA^{2}\right)$} \\
\hline protein & & & & 20.3 & 26.9 \\
\hline solvent & & & & 28.5 & 31.9 \\
\hline ligand $\left(\mathrm{PO}_{4}, \mathrm{ADP}\right)$ & & & & 18.2 & 46.7 \\
\hline \multicolumn{6}{|l|}{ Ramachandran analysis (\%) } \\
\hline most favored regions & & & & 95.6 & 95.6 \\
\hline additionally allowed regions & & & & 4.4 & 4.4 \\
\hline
\end{tabular}

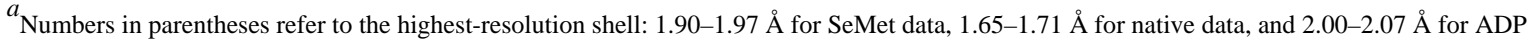
complex data.

${ }^{b} R_{\text {Sym }}=\Sigma|I-\langle I\rangle| \sigma I$, where $I$ is the observed intensity and $\langle I\rangle$ is the average intensity of multiple symmetry-related observations of that reflection.

${ }^{c}\langle I\rangle /\langle\sigma(I)\rangle$ is the rms value of the intensity measurements divided by their estimated standard deviation.

$d_{R_{\mathrm{Work}}}=\Sigma h k l|| F_{\mathrm{O}}|-| F_{\mathrm{C}} \| / \Sigma_{h k l}\left|F_{\mathrm{O}}\right|$, where $F_{\mathrm{O}}$ and $F_{\mathrm{C}}$ are the observed and calculated structure factors, respectively, for the $95 \%$ of the data used in refinement.

${ }^{e} R_{\text {free }}$ is calculated as described for $R_{\text {Work }}$ with $5 \%$ of the data excluded from refinement. 